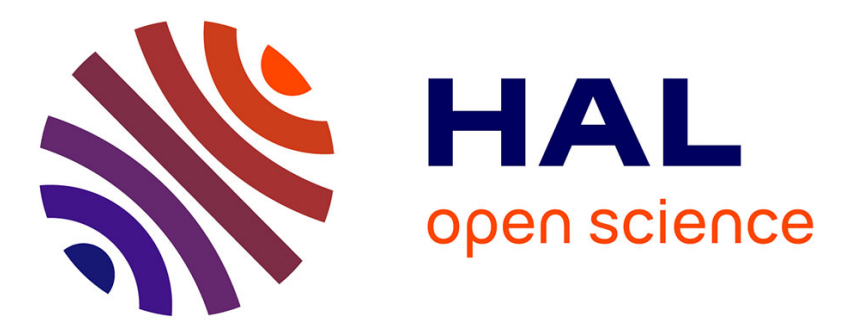

\title{
Dynamics of propagating front into sand ripples under regular waves
}

Julie Lebunetel-Levaslot, Armelle Jarno-Druaux, Alexander Ezersky, François

Marin

\section{- To cite this version:}

Julie Lebunetel-Levaslot, Armelle Jarno-Druaux, Alexander Ezersky, François Marin. Dynamics of propagating front into sand ripples under regular waves. Physical Review E: Statistical, Nonlinear, and Soft Matter Physics, 2010, 82 (3), 10.1103/PhysRevE.82.032301 . hal-02095110

\section{HAL Id: hal-02095110 https://hal.science/hal-02095110}

Submitted on 10 Apr 2019

HAL is a multi-disciplinary open access archive for the deposit and dissemination of scientific research documents, whether they are published or not. The documents may come from teaching and research institutions in France or abroad, or from public or private research centers.
L'archive ouverte pluridisciplinaire HAL, est destinée au dépôt et à la diffusion de documents scientifiques de niveau recherche, publiés ou non, émanant des établissements d'enseignement et de recherche français ou étrangers, des laboratoires publics ou privés. 
16 Pattern formation on a bottom under the action of surface 17 waves has been investigated theoretically and experimentally 18 for many years. The morphological characteristics of sand 19 ripple patterns observed in the near shore region are impor20 tant for the prediction of the dissipation of waves energy, and 21 for the sediment transport. Ripples also influence the biologi22 cal processes occurring on the bottom and the dispersion of 23 pollutants. Ayrton [1] and Bagnold [2] carried out the pio24 neering works on these structures. Detailed investigations of 25 the onset of instability caused by oscillating water over sand 26 were performed in $[3,4]$. The formation of vortices at the lee 27 side of the ripple crest and their ejection upward at flow 28 reversal were considered in $[5,6]$. These vortices control the 29 mass transfer between neighboring ripples during their for30 mation [6,7], and the wavelength of fully developed ripples 31 is proportional to the amplitude of the oscillatory flow $[6,8]$. 32 The stability of bottom patterns in relation to changes of 33 amplitude and frequency of water oscillations was studied in 34 [9].

35 In this paper, we focus on the investigation of the ampli36 fication of initial perturbations of small amplitude leading to 37 the formation of sand ripples. The front propagation plays a $38 \mathrm{key}$ role in the involved processes, and we present in this 39 brief report a detailed investigation of the characteristics of 40 this front. The study of front propagation has been consid41 ered in different unstable systems [10], and in particular in 42 numerous hydrodynamic systems [11-13]. The phenomenon 43 of front propagation for sand ripples under waves was men44 tioned in [2]. However, this propagation has not been accu45 rately investigated to our knowledge. The aim of the present 46 paper is to study the features of front propagation in sand 47 ripple patterns. The main difference between sand ripples 48 and the systems considered in [11-13] is the following. The 49 action of regular surface waves (propagating usually in one 50 direction) results in anisotropy of the sand ripples instability, 51 and consequently of the front propagation velocity, whereas 52 the systems studied in [11-13] may be considered as isotro53 pic. The characteristics of fronts propagating in the same

\footnotetext{
*Corresponding author. francois.marin@univ-lehavre.fr
}

direction as surface waves or in the opposite direction are 54 investigated in detail in the present study.

\section{EXPERIMENTAL SETUP AND RESULTS}

The experiments were performed in a $10 \mathrm{~m}$ long, $0.5 \mathrm{~m} 57$ high, and $0.49 \mathrm{~m}$ wide wave flume at Le Havre University. 58 Regular surface waves were produced by an oscillating 59 paddle at one end of the flume. At the other end a porous 60 beach was installed to minimize wave reflection. The tempo- 61 ral evolution of the free surface was measured with two fixed 62 resistive probes and analyzed with Goda's method [14]. The 63 reflection coefficient was less than $5 \%$ for all of the tests. 64 The mean water depth at rest was $d_{*}=27 \mathrm{~cm}$. Experiments 65 were carried out in a large range of wave and sediment 66 parameters: $0.95 \mathrm{~s} \leq T \leq 2.2 \mathrm{~s}, \quad 0.045 \mathrm{~m} \leq H \leq 0.099 \mathrm{~m}, 67$ $111 \mu \mathrm{m} \leq d_{50} \leq 375 \mu \mathrm{m}$, where $T$ and $H$ are the wave pe- 68 riod and height, respectively, and $d_{50}$ the median grain size. 69 For each test, the bed was initially flat and covered by a 2570 $\mathrm{mm}$ sand layer. In spite of the care taken to flatten the bot- 71 tom, some defects of flatness are observed. The maximum 72 amplitude of perturbations is approximately $2 \mathrm{~mm}$. The bed 73 deformation was measured from the first excitation cycles 74 with an optical method, detailed in [15]. The spatial reso- 75 lution in the horizontal and vertical directions was $0.5 \mathrm{~mm} / 76$ pixel. The dimensions of the processed field were $5.46 \mathrm{~m} 77$ long and $0.325 \mathrm{~m}$ width. The ripples wavelengths at the equi- 78 librium state were in the range $28.4 \mathrm{~mm} \leq \lambda \leq 77 \mathrm{~mm}$ for 79 present tests. Characteristics of ripples were obtained using 80 the one-dimensional (1D) -Hilbert transform. A great advan- 81 tage of this technique is that in each patch the amplitude and $\mathbf{8 2}$ phase of ripples may be determined. 83

Two distinct modes of ripple patterns formation are ob- 84 served. In the first mode, any perturbation on the bottom is 85 enough to trigger ripple formation and ripples form uni- 86 formly on the whole bed. In the second mode, patterns form 87 from isolated rippled zones (described as patches by Faraci 88 and Foti [16]). For present tests, patches appeared in zones 89 where the characteristic amplitude of disturbances was 90 greater or equal to the critical value of $2 \mathrm{~mm}$. Two nondi- 91 mensional parameters were used to characterize the regime 92 of pattern formation: the Reynolds number $\mathrm{Re}$ and the 93 Froude number Fr. These parameters are defined as follows: 94 


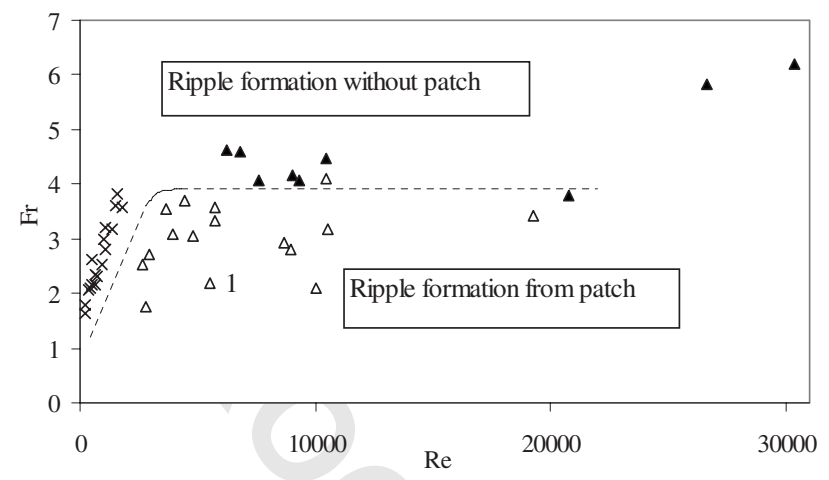

$\times$ Jarno-Druaux et al., $2004 \Delta$ Present tests, patch $\Delta$ Present tests, without patch

FIG. 1. Delineation of the two observed modes of ripple formation in the (Re,Fr) plane, indicating the boundary (dashed line) between the modes with and without patch (the test for which $\mathrm{Re}$ $=5512$ and $\mathrm{Fr}=2.2$ is identified with the label 1 ).

$95 \operatorname{Re}=U_{\infty} b / \nu, \operatorname{Fr}=U_{\infty} / \sqrt{(s-1) g d_{50}}$, where $b$ and $U_{\infty}$ are the 96 fluid particle semiexcursion and the fluid velocity amplitude 97 at the edge of the bed boundary layer, respectively, $s$ is the 98 relative density of sediment, $g$ the acceleration due to grav99 ity, and $\nu$ the water kinematic viscosity. The delineation of 100 the two observed modes of ripple formation in the (Re,Fr) 101 plane is presented in Fig. 1. The data of Jarno-Druaux et al. 102 [17] obtained in the same wave flume with lightweight grains 103 of relative density 1.35 and median grain diameter $170 \mu \mathrm{m}$ 104 are also shown in this figure. Present tests show that for fixed 105 values of $\mathrm{Re}$, there is a critical value $\mathrm{Fr}_{c r}$ of the Froude 106 number below which ripples form from localized sites; for $107 \mathrm{Fr}>\mathrm{Fr}_{c r}$, no "patch" is observed. The critical Froude number 108 becomes independent of the Reynolds number for $\mathrm{Re}$ $109>5000$. This suggests that for $\mathrm{Fr}_{c r}=\mathrm{Fr}_{c r_{-} \max } \approx 3.9$, the sedi110 ments move all over the bottom with a very low resistance to 111 motion for every hydrodynamic conditions and ripples can 112 form everywhere on the bottom. When $\mathrm{Fr}<\mathrm{Fr}_{c r_{\text {_max }}}$, the in113 ertial effects become more important for increasing values of $114 \mathrm{Re}$ (keeping constant the value of Fr), and the bed local 115 perturbations lead to patches formation. The critical value $116 \mathrm{Fr}_{c r}$ grows with $\mathrm{Re}$ when $\mathrm{Fr}<\mathrm{Fr}_{c r_{-} \text {max }}$. This may result from 117 a decrease of the gravity effects in comparison with the in118 ertial effects acting on the grains for increasing values of $\mathrm{Fr}$ 119 for a given value of $\operatorname{Re}(<5000)$, leading to a higher mobil120 ity of the grains and preventing patch formation.

121 In order to study the front propagation, we focus on a test 122 for which a slow dynamics of pattern formation from ampli123 tude defects is observed. We have $\mathrm{Re}=5512$ and $\mathrm{Fr}=2.2$ for 124 this test, and the mean ripple wavelength is $\lambda=42 \mathrm{~mm}$ at the 125 equilibrium state. An example of bed image in gray levels is 126 given in Fig. 2 for $n=800$ excitation cycles where three main 127 patches are clearly identified (P1 to P3). The temporal evo128 lution of the bottom elevation is plotted as a function of the

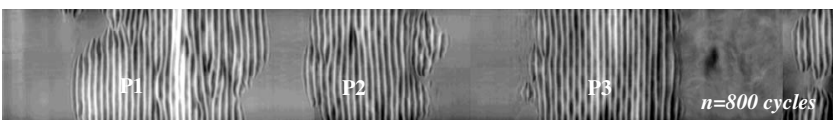

FIG. 2. Example of bed image in gray levels for $n$ $=800$ cycles $(\operatorname{Re}=5512, \mathrm{Fr}=2.2)$. P1 to P3 refer to the three processed patches.

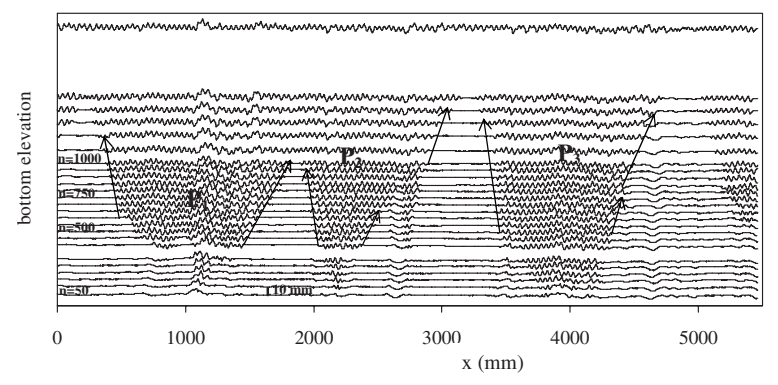

FIG. 3. Bottom elevation as a function of the $x$-longitudinal position and number of excitation cycles $(\operatorname{Re}=5512, \mathrm{Fr}=2.2)$. The arrows show the ripple front positions detected for the three patches $\mathrm{P} 1, \mathrm{P} 2$, and $\mathrm{P} 3$.

$x$-longitudinal position in Fig. 3, for $y=0.20 \mathrm{~m}$ where the $y 129$ axis refers to the cross-section direction. The origin of the $x 130$ axis is situated at $2.3 \mathrm{~m}$ from the wave paddle, increasing 131 values of $x$ corresponding to shorter distances from the ab- 132 sorbing beach. The time step is equal to 50 cycles for the first 133 1000 cycles, and afterward to 100 cycles. Isolated systems of 134 propagating ripples can be observed during more than 1000135 cycles before the invasion of the whole bottom. The estima- 136 tion of front velocities can then be performed on a long time 137 for the three observed patches. The bottom elevation signal 138 $\eta(x, t)$ of each patch is cut into two parts in order to process 139 the two fronts separately. The Fourier spectra of signals are 140 then calculated and harmonics are filtered. After this filtering 141 process, we get $\eta(x, t)=\eta_{m}(x, t) \cos [k x+\varphi(x, t)]$, where 142 $\eta_{m}(x, t)$ is for the slow varying amplitude, $\varphi(x, t)$ is the slow 143 varying phase of the bottom profile, and $k$ is the bottom wave 144 number.

Using the Hilbert transform,

$\hat{\eta}(x, t)=\frac{1}{\pi} P V\left[\int_{-\infty}^{+\infty} \frac{\eta(x, t)}{x-\chi} d \chi\right]=\eta_{m}(x, t) \sin [k x+\varphi(x, t)]$

where $P V$ denotes principal value, it is possible to determine 148 the phase and amplitude of sand ripples on the flume bottom 149 and compare with the theoretical predictions. We can con- 150 sider the bottom profile as the real part of a complex function 151 $\eta(x, t)=\operatorname{Re}\{A(x, t) \exp [i(k x)]\}$ with $A(x, t)=|A(x, t)| e^{i \varphi(x, t)}$ and 152 where

$$
|A(x, t)|=a=\sqrt{\eta^{2}(x, t)+\hat{\eta}^{2}(x, t)}, \quad \varphi(x, t)=\arctan \left(\frac{\hat{\eta}}{\eta}\right)-k x .
$$

We extract the module of the complex amplitude $a$ and the 155 phase $\varphi(x, t)$ from the signals. An example of the spatial 156 dependence for $a(x)$ and $\varphi(x)$ is shown in Fig. 4. It is impor- 157 tant to emphasize that large changes of phase occur at wave 158 front. The wave front is localized in the region where a tran- 159 sition from a low amplitude to a high (nearly constant) value 160 is detected. We have chosen the following criterion to deter- 161 mine the front position: the front is situated in the region 162 where the value of the amplitude is equal to $15 \%$ of the 163 maximum value for the patch. The ripple fronts are presented 164 in Fig. 3. They propagate linearly with time, and a good 165 coefficient of regression (in the range 0.70-0.98) is obtained. 166 The up-flow $\left(v_{p-}\right.$; propagation in the direction opposite to the 167 


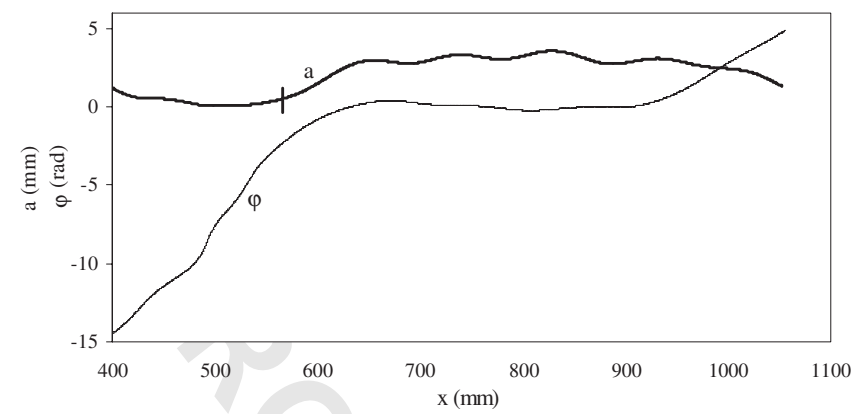

FIG. 4. Example of spatial distribution for the amplitude (bold line) and phase (thin line) of sand ripples for a front propagating upward (Patch P1, $t=550$ wave excitation cycles). The segment crossing the bold line delineates the border between the flat bottom and the ripple patch according to the criterion of threshold amplitude.

168 surface waves propagation) and down-flow ( $v_{p+}$; propagation 169 in the same direction as the surface waves) front velocities of 170 the patches are given in Table I. The results show that the 171 fronts propagating in the direction of surface wave propaga172 tion have greater velocities than the fronts propagating in the 173 opposite direction $\left(\left|v_{p+}\right|>\left|v_{p-}\right|\right)$.

\section{DISCUSSION OF RESULTS}

175 According to the present experimental results, the front 176 propagation may be considered as an envelope wave, and we 177 have found the amplitude and phase of this envelope. Let us 178 compare the experimental results with the solution of an 179 equation describing the envelope amplitude, the complex 180 Ginzburg-Landau equation (GLE) which is widely used to 181 investigate pattern dynamics [18]. Present experiments show 182 us that there is a threshold value of the initial bed perturba183 tions in the front propagation regime: perturbations with an 184 amplitude less than a critical value decay with time, whereas 185 perturbations with an amplitude greater than the critical 186 value grow. To take this effect into account, it is necessary to 187 keep the nonlinear terms proportional to the third and fifth 188 degrees of amplitude in the GLE (quintic version of GLE) 189 [10]. The cubic version of the GLE is able to describe the 190 linear instability of infinitely small perturbations and the 191 nonlinear amplitude saturation. The simplest model to de192 scribe the front propagation in our system is the following:

$193 \frac{\partial A}{\partial t}=\left(1+i c_{1}\right) \frac{\partial^{2} A}{\partial x^{2}}+\varepsilon A+\left(1+i c_{3}\right)|A|^{2} A-\left(1-i c_{5}\right)|A|^{4} A$

194 where $A$ is the complex amplitude of sand ripples, $\varepsilon$ the 195 super criticality $\left(\varepsilon<0\right.$ in our case), and $c_{1}, c_{3}, c_{5}$ are real

TABLE I. Up-flow and down-flow patch velocities for the three patches $\mathrm{P} 1, \mathrm{P} 2$, and $\mathrm{P} 3(\mathrm{Re}=5512, \mathrm{Fr}=2.2)$.

\begin{tabular}{ccc}
\hline \hline Patch & $\begin{array}{c}\text { Up-flow patch } \\
\text { velocity } v_{p-} \\
\left(\mathrm{mm} \mathrm{s}^{-1}\right)\end{array}$ & $\begin{array}{c}\text { Down-flow patch } \\
\text { velocity } v_{p+} \\
\left(\mathrm{mm} \mathrm{s}^{-1}\right)\end{array}$ \\
\hline 1 & -0.23 & 0.62 \\
2 & -0.19 & $0.64(400<n<650) ; 0.43(1000<n<1400)$ \\
3 & -0.16 & $0.37(500<n<800) ; 0.52(900<n<1400)$ \\
\hline \hline
\end{tabular}

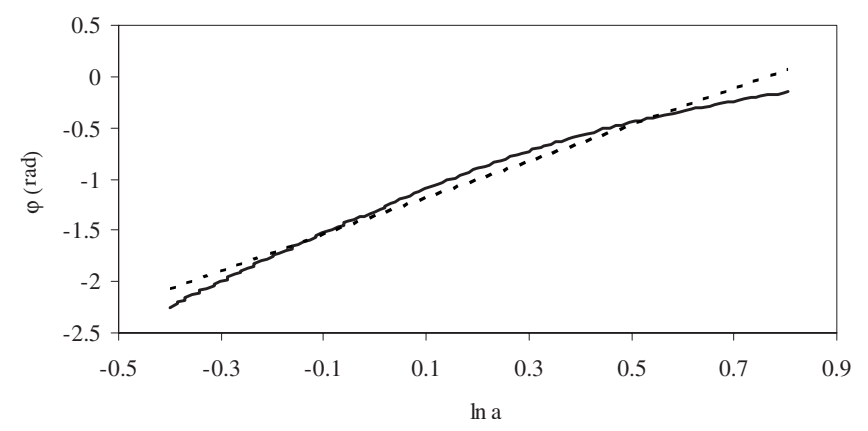

FIG. 5. Dependence of the wave phase $\varphi$ on the logarithm of wave amplitude ln $a$ for $462 \mathrm{~mm}<x<520 \mathrm{~mm}$ (bold curve) with its best linear fit approximation $(\varphi=1.82 \ln a-1.52$; square of the correlation coefficient $R^{2}=0.96$; dashed line); $t=700$ wave excitation cycles.

coefficients for dispersion $\left(c_{1}\right)$, cubic nonlinearity $\left(c_{3}\right)$, and 196 quintic nonlinearity $\left(c_{5}\right)$. The analytical solution of Eq. (3) 197 has the following form [19]: $A=e^{-i \Omega t} a(\xi) e^{i \phi(\xi)}, \quad \xi=x \mp V t 198$ where $V$ is the front velocity and $\Omega$ the frequency of sand 199 ripples. The amplitude and phase obey the following differ- 200 ential equations (anzatz): $\partial a / \partial \xi=K_{L \mp} a\left(1-a^{2}(\xi) / a_{N}^{2}\right), 201$ $\partial \phi / \partial \xi=q_{L}+\left(q_{N}-q_{L}\right) a^{2}(\xi) / a_{N}^{2}$. For propagating fronts, the 202 solution has the following form: 203

$$
a=a_{N} e^{K_{L \mp} \xi} / \sqrt{1+e^{2 K_{L \mp} \xi}} .
$$

The amplitude grows exponentially from an infinitely 205 small value to a constant value $a_{N}$. The six constants $K_{L \mp}, q_{L}, 206$ $q_{N}, \Omega, V, a_{N}$ are determined by inserting the ansatz into 207 Eq. (3) [10]. The sign "+" corresponds to a front which 208 propagates in the positive direction (direction of surface 209 waves propagation), $K_{L+}<0, \quad a(x=-\infty, t=0)=a_{N}, \quad a(x 210$ $=+\infty, t=0)=0$, and the sign "-" corresponds to a front 211 propagating in the opposite direction: $K_{L_{-}}>0, a(x=-\infty, t 212$ $=0)=0, a(x=+\infty, t=0)=a_{N}$. It should be noted that for re- 213 gions where $a=a_{N}$, we have $\phi=q_{N}(x \mp V t)$, and for $a \ll a_{N}, 214$ $\phi \cong q_{L}(x \mp V t)$. The profile of the sandy bottom may be 215 written as $\eta(x, t)=\operatorname{Re}[A(x, t) \exp (i k x)]=a(x \mp V t) \cos (\Omega t 216$ $\left.-q_{N, L}(x \mp V t)-k x\right)$. This means that $q_{L}$ and $q_{N}$ may be con- 217 sidered as infinitesimal and finite amplitude additional terms 218 for the wave number of sand ripples, respectively. Using the 219 $a_{N}^{2}$ expression from the first equation of ansatz, we find a 220 correlation between the phase and amplitude derivatives: 221 $\partial \phi / \partial \xi=q_{N}-\left[\left(q_{N}-q_{L}\right) \partial a / \partial \xi\right] / a K_{L \pm}$, and after integration we 222 get

$$
\phi=q_{N} \xi-\left[\left(q_{N}-q_{L}\right) / K_{L \mp}\right] \ln a .
$$

Excluding a linear growth of the phase with space for a given 225 instant, we are able to present a local correlation between the 226 wave amplitude $a(x)$ and the wave phase $\varphi(x): \varphi(x)=\left(q_{L} 227\right.$ $\left.-q_{N}\right) \ln a / K_{L_{ \pm}}$. Such correlation really occurs for the wave 228 front in sand ripples. Figure 5 shows an example of the 229 variation of the phase $\varphi$ with the amplitude $a$. Using the best 230 linear fit approximation (dashed line in Fig. 5), we deter- 231 mined the coefficient $\kappa_{ \pm}=\left(q_{L}-q_{N}\right) / K_{L \pm}$. The results are dif- 232 ferent for the fronts propagating in the direction of surface 233 waves and in the opposite direction. The values of the coef- 234 ficient $\kappa_{ \pm}$are estimated for both fronts of Patch 1, for dif- 235 ferent numbers of excitation cycles; these values are given in 236 
237 Table II. It can be noted that the linear dependence between 238 the phase $\varphi$ and $\ln a$ is obtained with lower values of the 239 regression coefficient for fronts propagating in the direction 240 of surface waves (except when $t=950$ excitation cycles). In 241 this case, for some instants, no estimation of $\kappa_{+}$could be 242 proposed. The fronts propagating in the direction of surface 243 waves are then not as regular as the fronts propagating in the 244 opposite direction. We were able to estimate the coefficient $245 K_{L_{ \pm}}$: the solution (4) shows us that this exponent may ap246 proximate the amplitude growth on the wave front. Using an 247 exponential approximation of experimental data, we have 248 found different coefficients for the fronts: the averaged value 249 for $K_{L+}$ was $K_{L+}=-0.047 \mathrm{~mm}^{-1}$, and for the front propagat$250 \mathrm{ing}$ in the opposite direction, $K_{L_{-}}=0.03 \mathrm{~mm}^{-1}$. The front 251 propagating in the direction of surface waves is "steeper" 252 than the front propagating in the opposite direction. We have 253 estimated the changes in wave number due to the finite am254 plitude of sand ripples: $q_{L}-q_{N} \approx 0.039 \mathrm{~mm}^{-1}$ for waves co255 directed with the surface waves, and $q_{L}-q_{N} \approx 0.025 \mathrm{~mm}^{-1}$ 256 for waves propagating in the opposite direction. In both 257 cases, the finite amplitude leads to a decrease in wave num258 ber $k+q_{N}$ in comparison with the linear wave number $k+q_{L}$, 259 but this effect is larger for the front propagating in the direc260 tion of surface waves.

261 The differences between the characteristics of the fronts 262 propagating in the same direction as the surface waves and in 263 the opposite direction may be due to the drift induced by 264 surface waves. It is well known [20] that in the bed boundary 265 layer above a flat bed, induced flows lead to mass transport 266 in the direction of waves propagation. Above sand ripples, 267 the momentum transfer and suspended sediment dynamics 268 are dominated by the formation and shedding at flow reversal 269 of lee wake vortices [21]. Present data involve weakly asym270 metrical waves $\left(B<0.1\right.$ where $B=3 b k_{s w} / 4 \sinh ^{2}\left(k_{s w} d_{*}\right)$ and $271 k_{s w}$ is the surface wave number). Using a one-dimensional 272 vertical (1DV) two-layer model where vortex shedding is 273 represented in the lower layer by a time-varying eddy vis274 cosity, Davies and Thorne [21] have shown that the near-bed 275 sand transport is in the direction of surface waves propaga276 tion for weakly asymmetrical waves. Such transport of sand 277 increases the front velocity $v_{p+}$ and decreases the velocity $278 v_{p-}$.

304

305

306 [1] H. Ayrton, Proc. R. Soc. London, Ser. A 84, 285 (1910).

307 [2] R. A. Bagnold, Proc. R. Soc. London, Ser. A 187, 1 (1946).

308 [3] P. Blondeaux, J. Fluid Mech. 218, 1 (1990).

309 [4] E. Foti and P. Blondeaux, Coastal Eng. 25, 227 (1995).

310 [5] V. Marieu et al., J. Geophys. Res. 113, C09007 (2008).

311 [6] K. H. Andersen et al., Phys. Rev. E 63, 066308 (2001).

312 [7] E. K. O. Hellén and J. Krug, Phys. Rev. E 66, 011304 (2002).

313 [8] T. Schnipper et al., Phys. Rev. E 78, 047301 (2008).

314 [9] J. L. Hansen et al., Nature (London) 410, 324 (2001).

315 [10] W. van Saarloos, Phys. Rep. 386, 29 (2003).

316 [11] J. Fineberg and V. Steinberg, Phys. Rev. Lett. 58, 1332 (1987).

317 [12] J. Fineberg et al., Phys. Rev. A 41, 5743 (1990).

318 [13] M. Fermigier et al., J. Fluid Mech. 236, 349 (1992).
TABLE II. Values of the inclination coefficient for the upstream and downstream fronts and different numbers of excitation cycles (Patch P1). The linear regression coefficients are given in parentheses.

\begin{tabular}{ccc}
\hline \hline Number of excitation cycles & $\kappa_{-}=\frac{q_{L}-q_{N}}{K_{L-}}$ & $\kappa_{+}=\frac{q_{L}-q_{N}}{K_{L+}}$ \\
\hline 550 & $+1.30\left(\mathrm{R}^{2}=0.99\right)$ & \\
600 & $+0.33\left(\mathrm{R}^{2}=0.99\right)$ & \\
650 & $+0.45\left(\mathrm{R}^{2}=0.99\right)$ & $-1.20\left(\mathrm{R}^{2}=0.95\right)$ \\
700 & $+1.80\left(\mathrm{R}^{2}=0.97\right)$ & \\
750 & $+0.66\left(\mathrm{R}^{2}=0.99\right)$ & $-0.12\left(\mathrm{R}^{2}=0.75\right)$ \\
800 & $+1.80\left(\mathrm{R}^{2}=0.99\right)$ & $-0.49\left(\mathrm{R}^{2}=0.81\right)$ \\
850 & $+0.60\left(\mathrm{R}^{2}=0.99\right)$ & $-0.51\left(\mathrm{R}^{2}=0.85\right)$ \\
900 & $+0.31\left(\mathrm{R}^{2}=0.94\right)$ & $-2.30\left(\mathrm{R}^{2}=0.93\right)$ \\
950 & $+0.28\left(\mathrm{R}^{2}=0.87\right)$ & $-0.33\left(\mathrm{R}^{2}=0.98\right)$ \\
\hline \hline
\end{tabular}

\section{CONCLUSIONS}

We have shown that depending on the values of the con- 280 trol parameters (Froude and Reynolds numbers), sand ripples 281 on the bottom may arise as a result of two types of bifurca- 282 tion: spatially homogeneous growth of small perturbations, 283 and appearance of patches. In the last case, wave front propa- 284 gation occurs. Using the Hilbert transform, we measured the 285 amplitude and phase of ripple waves, and we have found 286 coincidences between the experimental characteristics of 287 propagating fronts and the analytical solution of van Saar- 288 loos and Hohenberg [19]. Such coincidences allowed us to 289 find a correlation between the sand ripples amplitude and 290 wave number, and conclude that there exists an effect of 291 wave number decrease due to the finite amplitude of sand 292 ripples. Our measurements agree with the measurements of 293 other researchers (see, for example, [16]): the spatial period 294 of sand ripples increases with increasing ripples amplitude. 295 We have found that the propagating front characteristics de- 296 pend on the direction of surface waves which generate 297 ripples. If the front propagates in the direction of surface 298 waves, it has a larger celerity, is steeper and more irregular 299 than the front which propagates in the opposite direction. In 300 our opinion such differences are caused by the mean flow 301 induced by surface waves near the bottom.

302
[14] Y. Goda, Random Seas and Design of Marine Structures 319 (World Scientific, Singapore, 2000).

[15] F. Marin and A. B. Ezersky, Eur. J. Mech. B/Fluids 27, 251321 (2008).

[16] C. Faraci and E. Foti, Phys. Fluids 13, 1624 (2001). 322

[17] A. Jarno-Druaux et al., Eur. J. Mech. B/Fluids 23, 695 (2004). 324

[18] I. S. Aranson and L. Kramer, Rev. Mod. Phys. 74, 99 (2002). 325

[19] W. van Saarloos and P. C. Hohenberg, Phys. Rev. Lett. 64, 749326 (1990).

[20] M. S. Longuet-Higgins, Philos. Trans. R. Soc. London, Ser. A 328 245, 535 (1953).

329

[21] A. G. Davies and P. D. Thorne, J. Geophys. Res. 110, C05017 330 (2005). 